\title{
Slow relaxations and stringlike jump motions in fragile glass-forming liquids: Breakdown of the Stokes-Einstein relation
}

\author{
Takeshi Kawasaki and Akira Onuki \\ Department of Physics, Kyoto University, Kyoto 606-8502, Japan
}

(Dated: June 25, 2022)

\begin{abstract}
We perform molecular dynamics simulation on a glass-forming liquid binary mixture with the softcore potential in three dimensions. We investigate crossover of the configuration changes caused by stringlike jump motions. With lowering the temperature $T$, the motions of the particles composing strings become larger in sizes and displacements, while those of the particles surrounding strings become smaller. Then, the contribution of the latter to time-correlation functions tends to be longlived as $T$ is lowered. As a result, the relaxation time $\tau_{\alpha}$ and the viscosity $\eta$ grow more steeply than the inverse diffusion constant $D^{-1}$ at low $T$, leading to breakdown of the Stokes-Einstein relation. At low $T$, the diffusion occurs as activation processes and may well be described by short-time analysis of rare jump motions with broken bonds and large displacements. Some characteristic features of the van Hove self-correlation function arise from escape jumps over high potential barriers.
\end{abstract}

PACS numbers: 64.70.Q-,61.20.Lc,66.30.hh,66.30.-h

\section{INTRODUCTION}

Recently, much attention has been paid to the slow dynamics in glass-forming liquids [1, where the relaxation time $\tau_{\alpha}$ becomes extremely long and the viscosity $\eta$ grow dramatically with lowering the temperature $T$. In particular, it is well known experimentally that the curve of $\log \eta$ vs $1 / T$ (the Angell plot 2] ) becomes steeper with increasing $1 / T$ deviating from the Arrhenius behavior for fragile glass-forming liquids, while the Arrhenius behavior has been observed for strong glass formers like silica. However, it is not yet well understood how this dynamical crossover takes place microscopically for fragile glass formers. Hence, in this paper, we aim to investigate this aspect using molecular dynamics simulation.

In fragile glass-formers, stringlike motions of mobile particles [3 5] play a major role in the structural relaxation. The distribution of string lengths 4] was shown to widen with lowering $T$. However, the behaviors and the roles of stringlike motions have not yet been fully disclosed in the structural relaxation and the plastic deformations. In this paper, we analyze the configuration changes with the bond breakage theory [6, 7, which was originally used to detect the dynamic heterogeneity [8, 9] and has recently been generalized as a statisticalmechanical theory of irreversible particle rearrangements [10]. We then confirm that the configuration changes mostly occur as stringlike motions of mobile particles at low $T$. We shall furthermore see that the particles composing strings and those surrounding them behave differently depending on $T$ in a binary mixture at a fixed high density $n$ in three dimensions (3D). With lowering $T$, the former move over longer distances with multiple broken bonds, while the latter over shorter distances with a single broken bond. The number of the latter is several times larger than that of the former because of the large coordination numbers in $3 \mathrm{D}(\sim 10)$. Hence, the crossover of the latter motions with lowering $T$ should greatly influence the relaxation behavior of time-correlation functions and the relaxation time $\tau_{\alpha}$ at low $T$.

In liquid, the Stokes-Einstein relation $D \eta a / T=$ const. between the diffusion constant $D$ and the viscosity $\eta$ has been successfully applied even for a microscopic test particle diameter $a$. However, this relation is systematically violated in fragile supercooled liquids. 11, 12. Sillescu et al. 11] observed the power law behavior $D \propto \eta^{-\nu}$ with $\nu \cong 0.75$ at low $T$. The origin of this violation has often been ascribed to the dynamic heterogeneity without clear-cut calculations. A number of simulations have not clearly explained this violation [8, 13, 15,18. We shall see that the above-mentioned crossover of the particle motions around strings is the main origin of the violation for our fragile glass-forming system.

For sufficiently low $T$ in the glass-forming condition, the van Hove self-correlation function $G_{s}(r, t)$ tends to have a minimum at a particle length $r_{\mathrm{m}}$ and slowly grows in the outer region $r>r_{\mathrm{m}}$ forming secondary peaks 13 18. Here, $r$ represents the particle displacement length in a time interval of $t$. We shall see that its minimum value at $r=r_{\mathrm{m}}$ becomes very small at low $T$, indicating fast passage of the particles across potential barriers at $r \sim r_{\mathrm{m}}$. These behaviors reflect the fact that the diffusion arises from escape jumps from well-defined transient cages with long life times. Sastry et al. called this the landscape-dominated regime [14, which emerges on approaching the glass transition. We shall also see that the contribution to the mean square displacement $M(t)$ from the particles with large displacements behaves as $6 D t$ soon after the ballistic time region, where the thermal vibrational contributions from the other particles in the interior $r<r_{\mathrm{m}}$ are removed.

The organization of this paper is as follows. In Sec.II, our simulation method will be explained. In Sec.III, the bond-breakage theory in our previous work 6, 7, 10 will be used to examine the particle motions around strings for various $T$. In Sec.IV, we will examine the longdistance diffusion, which will turn out to be governed by the activated dynamics at low $T$. We will also exam- 
ine the van Hove self-correlation function $G_{s}(r, t)$ in the landscape-dominated regime.

\section{NUMERICAL METHOD}

In this paper we show results of molecular dynamics simulation of binary mixtures composed of two species, 1 and 2 , in $3 \mathrm{D}$ at low $T$. We impose the periodic boundary condition without applying shear flow. The composition is $c=N_{2} /\left(N_{1}+N_{2}\right)=0.5$ and the total particle number is $N=N_{1}+N_{2}=10^{4}$. The two species have different diameters $\sigma_{1}$ and $\sigma_{2}$ with $\sigma_{2} / \sigma_{1}=1.2$. The particles interact via the soft-core potential,

$$
v_{\alpha \beta}(r)=\epsilon\left(\frac{\sigma_{\alpha \beta}}{r}\right)^{12}-C_{\alpha \beta} \quad\left(r<r_{\text {cut }}\right),
$$

where $\alpha$ and $\beta$ represent the particle species $(\alpha, \beta=1,2)$, $r$ is the particle distance, and $\epsilon$ is the characteristic interaction energy. The interaction lengths are defined by

$$
\sigma_{\alpha \beta}=\left(\sigma_{\alpha}+\sigma_{\beta}\right) / 2
$$

The potential vanishes for $r>r_{\text {cut }}=3 \sigma_{1}$. The constants $C_{\alpha \beta}$ ensure the continuity of the potential at $r=r_{\text {cut }}$. The masses of the two species satisfy $m_{2} / m_{1}=\left(\sigma_{2} / \sigma_{1}\right)^{3}$. The average density is given by $n=N / V=0.8 \sigma_{1}^{-3}$ as in some previous papers 6, 17, 10, 15, 19, where $V$ is the system volume. The system length is $L=V^{1 / 3}=23.2 \sigma_{1}$. Space and time are measured in units of $\sigma_{1}$ and

$$
\tau_{0}=\sigma_{1} \sqrt{m_{1} / \epsilon}
$$

The temperature $T$ is measured in units of $\epsilon / k_{B}$.

We started from a liquid state at a high temperature, quenched the system to a final low temperature, and waited for a long time of order $10^{5}$. We imposed a NoseHoover thermostat in these steps. However, after this preparation of initial states, we removed the thermostat and integrated the Newton equations under the periodic boundary condition in the time range $t>0$. Thus, the particle numbers, the total volume, and the total energy are conserved in our simulation ( $N V E$ ensemble). At the lowest temperature $T=0.24$, the simulation time was $5 \times 10^{5}$ for each run and data were taken in the time range $5 \times 10^{4}<t<5 \times 10^{5}$, during which we did not detect any appreciable aging effects in various quantities such as the average potential energy and $F_{s}(q, t)$ defined below.

We are interested in supercooled states, where the structural relaxation time $\tau_{\alpha}$ is very long. In terms of the self part of the density time-correlation function,

$$
F_{s}(q, t)=\frac{1}{N} \sum_{j}\left\langle\exp \left[i \boldsymbol{q} \cdot \Delta \boldsymbol{r}_{j}\left(t_{0}, t_{0}+t\right)\right]\right\rangle,
$$

$\tau_{\alpha}$ is usually defined at $q=2 \pi \sigma_{1}^{-1}$ by

$$
F_{s}\left(q, \tau_{\alpha}\right)=e^{-1}
$$

Here, $\boldsymbol{q}$ is the wave vector with $q=|\boldsymbol{q}|$ and $\Delta \boldsymbol{r}_{j}\left(t_{0}, t_{1}\right)=$ $\boldsymbol{r}_{j}\left(t_{1}\right)-\boldsymbol{r}_{j}\left(t_{0}\right)$ is the displacement vector of particle $j$. In this paper, $\langle\cdots\rangle$ denotes taking the average over the initial time $t_{0}$ of the time interval and over several simulation runs. This is needed for averaging quantities involving two widely separated times, for which accurate results do not follow only by the average over all the particles for our system size.

It is worth noting that the stress time-correlation function behaves similarly to $F_{s}(q, t)$, both considerably decreasing in the early stage due to the thermal motions (the so-called $\beta$ relaxation) [6, 19]. Since the time integral of the stress time-correlation function is equal to the shear viscosity $\eta$ in the linear response regime, it is natural to expect weak $T$ dependence of the ratio $\eta / \tau_{\alpha}$. For the same soft-core potential (2.1), the following relation was previously obtained [15]:

$$
\eta \cong(2 \pi)^{-1} \sigma_{1}^{-3} k_{B} T \tau_{\alpha},
$$

in the range $1<\tau_{\alpha}<10^{4}$ at $n=0.8 \sigma_{1}^{-3}$.

\section{BOND BREAKAGE}

\section{A. Stringlike motions and their crossover}

We analyze the configuration changes in the bond breakage scheme [6, 7, 10]. They occur as jump motions causing irreversible particle rearrangements.

First, we introduce the concept of bond breakage. At an initial time $t=t_{0}$, two particles $i \in \alpha$ and $j \in \beta$ $(\alpha, \beta=1,2)$ are treated to be bonded if

$$
r_{i j}\left(t_{0}\right)<A_{1} \sigma_{\alpha \beta}
$$

Hereafter, $r_{i j}(t)=\left|\boldsymbol{r}_{i}(t)-\boldsymbol{r}_{j}(t)\right|$ is the distance between these particles at time $t$. At a later time $t=t_{1}>t_{0}$, this bond is treated to be broken if

$$
r_{i j}\left(t_{1}\right)>A_{2} \sigma_{\alpha \beta}
$$

Here, $A_{1} \sigma_{\alpha \beta}$ are slightly larger than the first peak distances of the pair correlation functions $g_{\alpha \beta}(r)$ and $A_{2}$ is somewhat larger than $A_{1}$. In this paper, we set $A_{1}=1.3$ and $A_{2}=1.7$. At high densities in $3 \mathrm{D}$, the bond number around each particle is of order 10 because it is on the order of the coordination number. Let $N_{b}\left(t_{0}\right)$ be the number of the bonds defined at time $t_{0}$ and $N_{b}\left(t_{0}, t_{0}+t\right)$ be the number of the unbroken bonds at later time $t_{0}+t$. Then, the fraction of the unbroken or surviving bonds after time $t$ is given by

$$
F_{b}(t)=\left\langle N_{b}\left(t_{0}, t_{0}+t\right) / N_{b}\left(t_{0}\right)\right\rangle
$$

The bond breakage time $\tau_{b}$ is determined by [6, 7]

$$
F_{b}\left(\tau_{b}\right)=e^{-1}
$$

Nonlinear rheology in supercooled states [7, 20, is governed by the bond breakage and is characterized by $\tau_{b}$. 


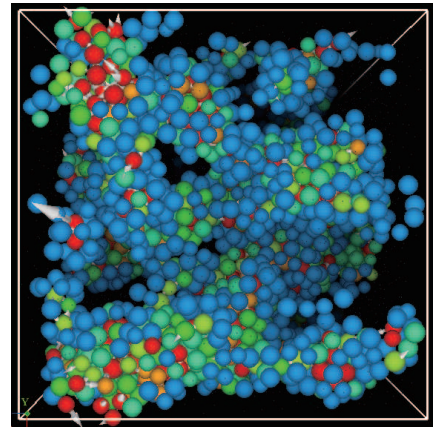

(b) $\mathrm{T}=0.24, \mathrm{~B}_{\mathrm{i}}^{>}>0$

$\mathrm{B}_{\mathrm{i}}$

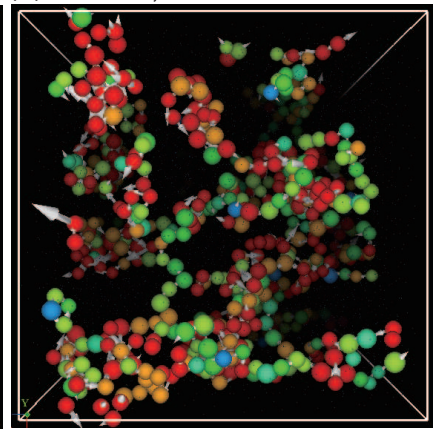

$\begin{array}{llllll}1 & 2 & 3 & 4 & 5 & 6\end{array}$

FIG. 1: (Color online) (a) $\boldsymbol{B}$ particles (those with $\mathcal{B}_{i}=$ $\left.\mathcal{B}_{i}\left(t_{0}, t_{1}\right)>0\right)$ composed of their strings and their neighbors. (b) $\boldsymbol{B} \boldsymbol{L}$ particles (those with $\mathcal{B}_{i}^{>}\left(t_{0}, t_{1}\right)>0$ ) composed of strings, which have large $\mathcal{B}_{i} \geq 3$ due to large displacements. These are snapshots at $t=t_{1}-t_{0}=10^{4} \cong 10^{-1} \tau_{\alpha}$ or at $\phi_{b}(t)=0.1$ for $T=0.24$ (see Table 1 ). The particle colors represent $\mathcal{B}_{i}$ according to the color bar below. Arrows represents $\Delta \boldsymbol{r}_{i}\left(t_{0}, t_{1}\right)$.

Furthermore, we introduce the broken bond number for each particle $i$ in terms of $A_{1}$ and $A_{2}$ as [10]

$$
\begin{aligned}
\mathcal{B}_{i}\left(t_{0}, t_{1}\right)= & \sum_{j} \theta\left(A_{1} \sigma_{\alpha \beta}-r_{i j}\left(t_{0}\right)\right) \\
& \times \theta\left(r_{i j}\left(t_{1}\right)-A_{2} \sigma_{\alpha \beta}\right),
\end{aligned}
$$

where $\theta(u)$ is the step function being zero for $u \leq 0$ and 1 for $u>0$. This number is a nonnegative integer, tending to zero as $t_{1} \rightarrow t_{0}$ from $A_{1}<A_{2}$ and increasing to $1,2, \cdots$ upon bond breakage. The particles with $\mathcal{B}_{i}\left(t_{0}, t_{1}\right)>0$ may be called $\boldsymbol{B}$ particles, while those with $\mathcal{B}_{i}\left(t_{0}, t_{1}\right)=0$ may be called non- $\boldsymbol{B}$ particles. The $\boldsymbol{B}$ particles are surrounded by different particle configurations at the initial and final times $t=t_{0}$ and $t_{1}$.

The displacements of the $\boldsymbol{B}$ particles may not be large if they are neighbors of those undergoing stringlike motions. Thus it is convenient to group the $\boldsymbol{B}$ particles further into those with large displacement $\Delta r_{i}>\ell_{m}$ and those with small displacement $\Delta r_{i}<\ell_{m}$, where $\ell_{m}$ is a minimum jump length and

$$
\Delta r_{i}=\Delta r_{i}\left(t_{0}, t_{1}\right)=\left|\boldsymbol{r}_{i}\left(t_{1}\right)-\boldsymbol{r}_{i}\left(t_{0}\right)\right|
$$

represents the displacement in time interval $\left[t_{0}, t_{1}\right]$. These particles are called $\boldsymbol{B} \boldsymbol{L}$ and $\boldsymbol{B} \boldsymbol{S}$ particles, respectively. Their broken bond numbers are written as

$$
\begin{aligned}
& \mathcal{B}_{i}^{>}\left(t_{0}, t_{1}\right)=\mathcal{B}_{i}\left(t_{0}, t_{1}\right) \theta\left(\Delta r_{i}\left(t_{0}, t_{1}\right)-\ell_{m}\right), \\
& \mathcal{B}_{i}^{<}\left(t_{0}, t_{1}\right)=\mathcal{B}_{i}\left(t_{0}, t_{1}\right) \theta\left(\ell_{m}-\Delta r_{i}\left(t_{0}, t_{1}\right)\right) .
\end{aligned}
$$

In this paper, we set $\ell_{m}=0.8$. A small change of its value does not essentially change our conclusions. See SubsecIVD and Fig.12 on this point.

In Fig.1a, we display only the $\boldsymbol{B}$ particles (about 600 ) at $t=t_{1}-t_{0}=10^{4} \cong 10^{-1} \tau_{\alpha}$ for $T=0.24$, where the
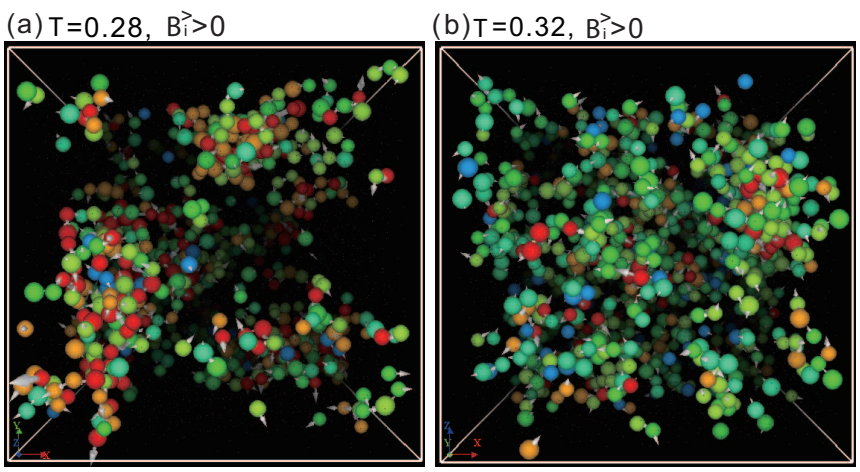

$\mathrm{B}_{\mathrm{i}}$

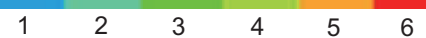

FIG. 2: (Color online) $\boldsymbol{B} \boldsymbol{L}$ particles (those with $\mathcal{B}_{i}^{>}\left(t_{0}, t_{1}\right)>$ 0 ) for (a) $T=0.28$ and $t=t_{1}-t_{0}=450$ and for (b) $T=0.32$ and $t=t_{1}-t_{0}=50$. The corresponding snapshot for $T=$ 0.24 is given in Fig.1b. Here, $t=t_{1}-t_{0}$ is determined from $\phi_{b}^{>}(t)=0.1$. The particle colors represent $\mathcal{B}_{i}$ according to the color bar.The strings become ill-defined with increasing $T$. Arrows represents $\Delta \boldsymbol{r}_{i}\left(t_{0}, t_{1}\right)$.

particle colors represent $\mathcal{B}_{i}=\mathcal{B}_{i}\left(t_{0}, t_{1}\right)$. We can see that many particles with $\mathcal{B}_{i}=1$ surround those with $\mathcal{B}_{i} \geq 2$. Here, the latter particles have undergone stringlike motions with large displacements $(\gtrsim 1)$. Thus, in Fig.1b, we depict only the $\boldsymbol{B} \boldsymbol{L}$ particles with $\Delta r_{i}>0.8$. Here, the average of $\Delta r_{i}$ over the $\boldsymbol{B} \boldsymbol{L}$ is 1.29 , while that over the $\boldsymbol{B} \boldsymbol{S}$ particles is 0.152 (see Fig.4 below). We also notice that a majority of the $\boldsymbol{B} \boldsymbol{L}$ particles ( $74 \%$ here) belong to the first (small) species (see Table 1 below). Thus, for this time elapse of $10^{4}$, the $\boldsymbol{B} \boldsymbol{L}$ and $\boldsymbol{B} \boldsymbol{S}$ particles mostly form strings and their neighbors, respectively.

At $T=0.24$, a large fraction of the $\boldsymbol{B} \boldsymbol{S}$ particles surrounding strings transiently moved over relatively large distances $(>0.5)$ at the string formation but eventually returned to their original positions (not shown in Fig.1b). Though rather rare, we also observed that some particles largely moved without string formation and returned to their original positions after some time (say, 400). These reversible jumps were observed by Vollmayr-Lee [21.

However, with increasing $T$, the difference between the $\boldsymbol{B} \boldsymbol{L}$ and $\boldsymbol{B} \boldsymbol{S}$ particles becomes less distinct. In Fig.2, we elucidate how the strings are changed at $T=0.28$ and 0.32 , where the number fraction of $\boldsymbol{B} \boldsymbol{L}$ particles $\phi_{b}^{>}$is 0.1 (see Eq.(3.10) below for its definition). As compared to the snapshot at $T=0.24$ in Fig.1b, they are shorter and more expanded with smaller $\mathcal{B}_{i}$, so they should rather be treated as clusters. This result is consistent with the previous calculation of the distributions of string lengths and cluster sizes for various $T$ by Donati et al 4 , 5. With increasing $T$, we also notice that the composition of the large particles increases among the $\boldsymbol{B} \boldsymbol{L}$ particles, leading to larger volume fractions of the $\boldsymbol{B} \boldsymbol{L}$ particles even at fixed $\phi_{b}^{>}=0.1$ (see Table 1 below). 

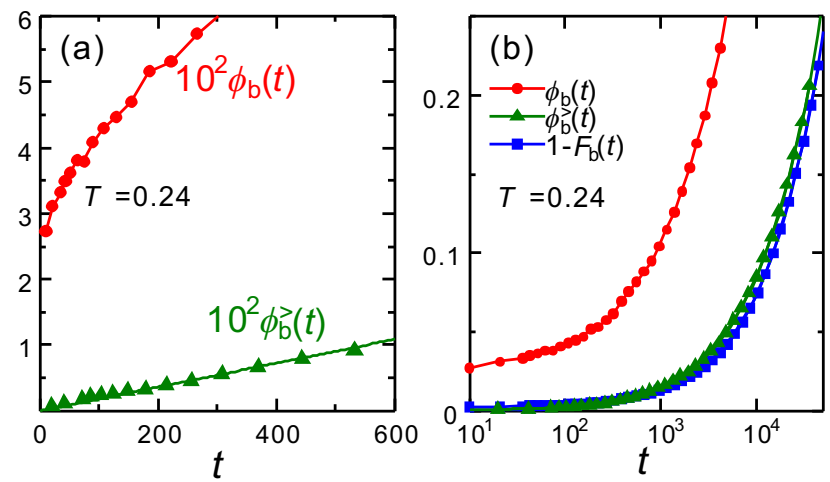

FIG. 3: (Color online) Fractions of the $\boldsymbol{B}$ and $\boldsymbol{B} \boldsymbol{L}$ particles, $\phi_{b}(t)$ and $\phi_{b}^{>}(t)$, respectively, for $T=0.24$. They are shown for $t<800$ (left) and on long times (right). Also displayed is the fraction of surviving bonds $1-F_{b}(t)$ nearly coinciding with $\phi_{b}^{>}(t)$ on long timescales (right).
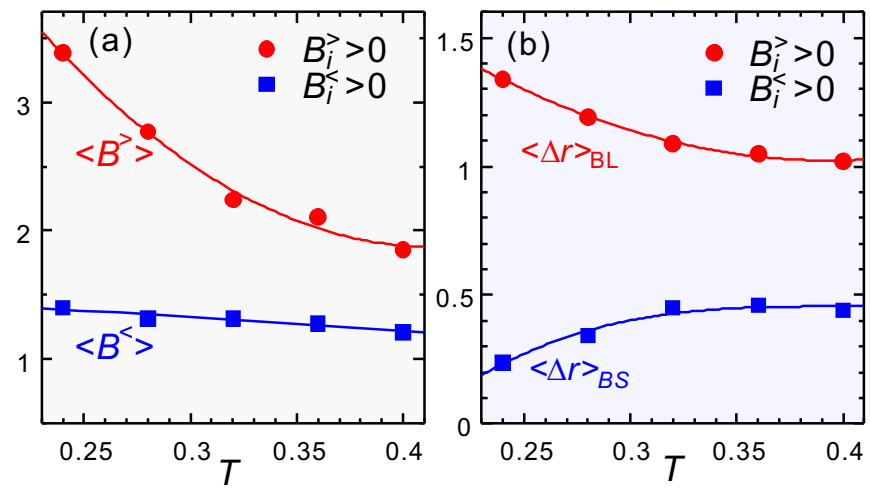

FIG. 4: (Color online) (a) Averages $\left\langle\mathcal{B}^{>}\right\rangle$and $\left\langle\mathcal{B}^{<}\right\rangle$in Eqs.(3.13) and (3.14) vs $T$. (b) Averages $\left\langle\Delta r_{B L}\right\rangle$ and $\left\langle\Delta r_{B S}\right\rangle$ in Eqs.(3.15) and (3.16) vs $T$. Here, $\phi_{b}^{>}(t)=0.1$, from which $t=t_{1}-t_{0}$ is determined for each $T$ as in Table 1 .

\section{B. Differences between $B L$ and $B S$ particles}

Let us consider the numbers of the $\boldsymbol{B}$ and $\boldsymbol{B} \boldsymbol{L}$ particles in the system. The average number fractions of these particles are given by

$$
\phi_{b}(t)=\frac{1}{N} \sum_{j}\left\langle\theta\left(\mathcal{B}_{j}\left(t_{0}, t_{1}\right)\right)\right\rangle,
$$

TABLE I: Data for five temperatures, where $t, \phi_{b}(t), c_{b}^{>}(t)$, and $c_{b}^{<}(t)$ are those at $\phi_{b}^{>}(t)=0.1$. See Fig.4 also. Here, $\phi_{b}(t)=\phi_{b}^{>}(t)+\phi_{b}^{<}(t)$ is the fraction of the $\boldsymbol{B}$ particles, while $c_{b}^{>}(t)$ and $c_{b}^{<}(t)$ are the compositions of the larger species among the $\boldsymbol{B} \boldsymbol{L}$ and $\boldsymbol{B} \boldsymbol{S}$ particles, respectively.

\begin{tabular}{|c||c|c||c|c|c|c|}
\hline$T$ & $\tau_{\alpha}$ & $\tau_{b}$ & $t$ & $\phi_{b}(t)$ & $c_{b}^{>}(t) c_{b}^{<}(t)$ & \\
\hline 0.24 & 109000 & 270000 & 10000 & 0.38 & 0.247 & 0.547 \\
\hline 0.28 & 1170 & 103000 & 450 & 0.46 & 0.309 & 0.496 \\
\hline 0.32 & 41.0 & 749 & 50 & 0.59 & 0.329 & 0.492 \\
\hline 0.36 & 11.4 & 267 & 20 & 0.61 & 0.339 & 0.481 \\
\hline 0.40 & 5.91 & 141 & 10 & 0.59 & 0.330 & 0.463 \\
\hline
\end{tabular}

$$
\phi_{b}^{>}(t)=\frac{1}{N} \sum_{j}\left\langle\theta\left(\mathcal{B}_{j}^{>}\left(t_{0}, t_{1}\right)\right)\right\rangle,
$$

where $t=t_{1}-t_{0}$. The $\phi_{b}(t)$ is the fraction of the particles with broken bonds, while $\phi_{b}^{>}(t)$ is that of the particles with broken bonds and large displacements. We may call $\phi_{b}^{>}(t)$ the string fraction also. In our previous paper [10, we have introduced a bond-preserving time $\tau_{\mathrm{bp}}$ by

$$
1-\phi_{b}\left(\tau_{\mathrm{bp}}\right)=e^{-1},
$$

which is the timescale of each particle to have a broken bond. For $T=0.24$, we have $\tau_{b}=2.7 \times 10^{5}=2.7 \tau_{\alpha}$ and $\tau_{\mathrm{bp}}=3.2 \times 10^{4}=0.32 \tau_{\alpha}$. Note that $\tau_{b}$ is longer than $\tau_{\mathrm{bp}}$ by one order of magnitude due to the large coordination number in $3 \mathrm{D}$.

In Fig. $3, \phi_{b}(t)$ and $\phi_{b}^{>}(t)$ are plotted on short and long timescales. Salient features are as follows. (i) First, on a microscopic timescale $(t \gtrsim 4), \phi_{b}(t)$ quickly approaches a small number 0.03 due to the thermal motions. (In $2 \mathrm{D}$, we have obtained an algebraic growth, $\phi_{b}(t) \sim t^{0.6}$, for small $t$, however [10.) (ii) Second, in the early stage $(t \lesssim 600), \phi_{b}^{>}(t)$ grows linearly as

$$
\phi_{b}^{>}(t) \cong \tau_{\mathrm{st}}^{-1} t
$$

The coefficient $\tau_{\mathrm{st}}^{-1}$ is the average frequency of rare jump motions per particle in the early stage $t \ll \tau_{\text {st }}$ (where $\left.\phi_{b}^{>}(t) \ll 1\right)$. Therefore, it is analogous to the nucleation rate in metastable systems [22]. In our case, $\tau_{\text {st }}$ is of order $\tau_{b}$ in Eq.(3.4). See Fig.5d for the $T$ dependence of $\tau_{\text {st }}$. (iii) Third, in the whole time range in Fig. $3, \phi_{b}(t)$ is much larger than $\phi_{b}^{>}(t)$. For $t \gg 1$, this is because several broken bonds are produced around a string as in Fig.1a. In Fig.3b, the ratio $\phi_{b}(t) / \phi_{b}^{>}(t)$ is of order 5 for $t \gtrsim 10^{3}$. In addition, $\phi_{b}^{>}(t)$ is close to the broken bond fraction $1-F_{b}(t)$, where $F_{b}(t)$ has appeared in Eq.(3.4). They are very close for $T \lesssim 0.3$ in the present case. (iv) Fourth, at long times $t \gtrsim 1000$, we have $\phi_{b}^{>}(t) \sim t^{0.76}$.

In Fig. 4, we show how $\boldsymbol{B} \boldsymbol{L}$ and $\boldsymbol{B} \boldsymbol{S}$ paricles behave differently. Displayed in the left are the averages of $\mathcal{B}_{i}\left(t_{0}, t_{1}\right)$ among the $\boldsymbol{B} \boldsymbol{L}$ and $\boldsymbol{B} \boldsymbol{S}$ particles. They are written as

$$
\begin{aligned}
\left\langle\mathcal{B}^{>}\right\rangle & =\frac{1}{\phi_{b}^{>} N} \sum_{i}\left\langle\mathcal{B}_{i}^{>}\left(t_{0}, t_{1}\right)\right\rangle, \\
\left\langle\mathcal{B}^{<}\right\rangle & =\frac{1}{\phi_{b}^{<} N} \sum_{i}\left\langle\mathcal{B}_{i}^{<}\left(t_{0}, t_{1}\right)\right\rangle,
\end{aligned}
$$

where $\phi_{b}^{<}=\phi_{b}-\phi_{b}^{>}$is the average fraction of the $\boldsymbol{B} \boldsymbol{S}$ particles. The average broken-bond number is 3.4 for the $\boldsymbol{B} \boldsymbol{L}$ particles and is 1.4 for the $\boldsymbol{B} \boldsymbol{S}$ particles at $\phi_{b}^{>}(t)=$ 0.1 for $T=0.24$. Displayed in the right are the averages of the displacements $\Delta r_{i}$ in Eq.(3.6) among the $\boldsymbol{B} \boldsymbol{L}$ and $\boldsymbol{B} \boldsymbol{S}$ particles,

$$
\begin{aligned}
\langle\Delta r\rangle_{B L} & =\frac{1}{\phi_{b}^{>} N} \sum_{i}\left\langle\Delta r_{i} \theta\left(\mathcal{B}_{i}^{>}\right)\right\rangle, \\
\langle\Delta r\rangle_{B S} & =\frac{1}{\phi_{b}^{<} N} \sum_{i}\left\langle\Delta r_{i} \theta\left(\mathcal{B}_{i}^{<}\right)\right\rangle,
\end{aligned}
$$



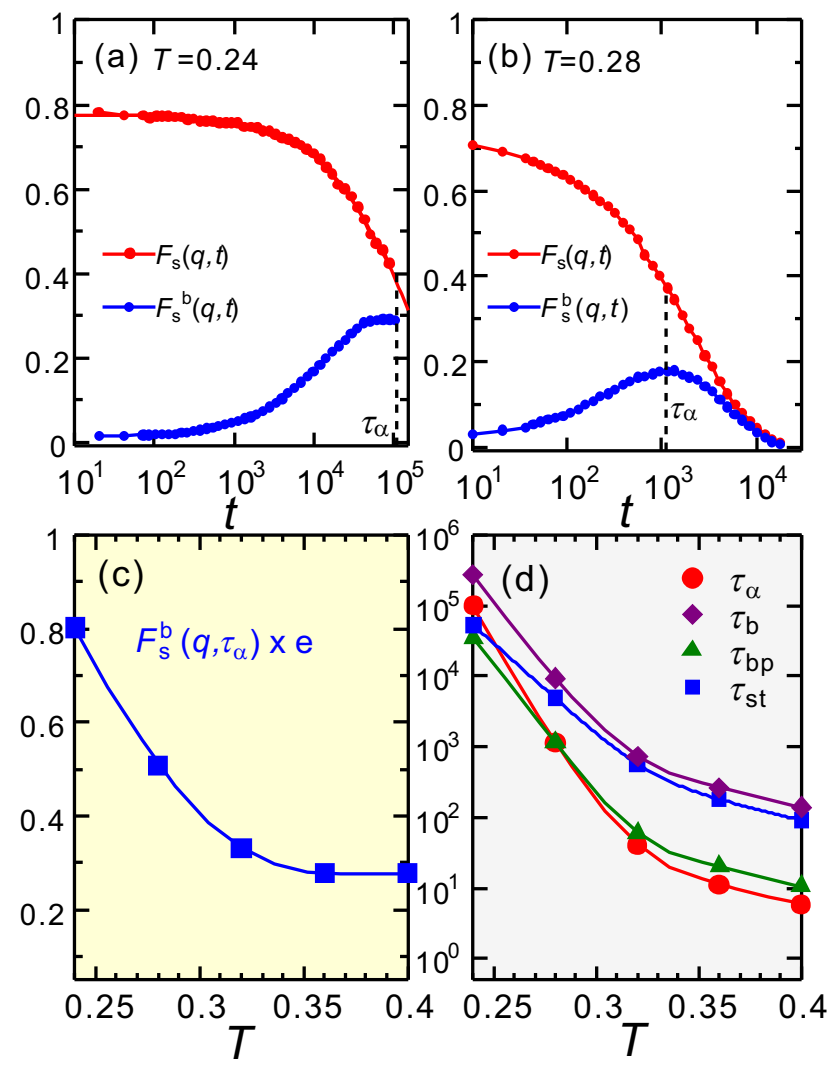

FIG. 5: (Color online) Top: $F_{s}(q, t)$ in Eq. $(2.4)$ and $F_{s}^{b}(q, t)$ in Eq.(3.17) for (a) $T=0.24$ and (b) $T=0.28$. Bottom: $F_{s}^{b}\left(q, \tau_{\alpha}\right)$ vs $T$ in (c) and three characteristic times $\tau_{\alpha}$ in Eq.(2.5), $\tau_{\text {bp }}$ in Eq.(3.11), $\tau_{\text {st }}$ in Eq.(3.12), and $\tau_{b}$ in Eq.(3.4) in (d). Here, $\tau_{\alpha}$ grows more steeply than the others at low $T$.

where $\Delta r_{i}, \mathcal{B}_{i}^{>}$, and $\mathcal{B}_{i}^{<}$are abbreviations of $\Delta r_{i}\left(t_{0}, t_{1}\right)$, $\mathcal{B}_{i}^{>}\left(t_{0}, t_{1}\right)$, and $\mathcal{B}_{i}^{<}\left(t_{0}, t_{1}\right)$, respectively. Remarkably, $\langle\Delta r\rangle_{B L}$ and $\langle\Delta r\rangle_{B S}$ are distinctly separated and $\ell_{\mathrm{m}}$ in Eqs.(3.7) and (3.8) has bee chosen to be 0.8 between them. Table 1 presents more data, where $c_{b}^{>}(t)$ and $c_{b}^{<}(t)$ are the compositions of the larger species among the $\boldsymbol{B} \boldsymbol{L}$ and $\boldsymbol{B} \boldsymbol{S}$ particles, respectively. The former is the composition within strings and is only 0.25 at $T=0.24$.

Thus the motions of the $\boldsymbol{B} \boldsymbol{L}$ and $\boldsymbol{B} \boldsymbol{S}$ particles (those composing strings and those surrounding strings) are increasingly different with lowering $T$. This should be a characteristic feature of fragile glass-forming liquids 2 , 12, 14, where the role of the thermal fluctuations in the configuration changes crosses over with varying $T$.

\section{Steep growth of $\tau_{\alpha}$ at low temperatures}

The physical meaning of $\tau_{\alpha}$ is not straightforward. First, $F_{s}(q, t)$ in Eq. (2.4) relaxes to a plateau value rapidly due to the thermal motions, which is about 0.8 for $T=0.24$ in Fig.5a and about 0.7 for $T=0.28$ in Fig.5b. Second, with lowering $T$, the motions of the $\boldsymbol{B} \boldsymbol{S}$ particles (those surrounding strings) become smaller as in Fig.4b, so their contribution to $F_{s}(q, t)$ becomes long-lived.

To examine the second feature in more detail, we define the conditional self-time-correlation function,

$$
F_{s}^{b}(q, t)=\frac{1}{N} \sum_{j}\left\langle\theta\left(\mathcal{B}_{j}\right) \exp \left[i \boldsymbol{q} \cdot\left(\Delta \boldsymbol{r}_{j}\right]\right\rangle\right.
$$

where $\mathcal{B}_{j}$ and $\Delta \boldsymbol{r}_{j}$ are abbreviations of $\mathcal{B}_{j}\left(t_{0}, t_{1}\right)$ and $\Delta \boldsymbol{r}_{j}\left(t_{0}, t_{1}\right)$, respectively, and only the $\boldsymbol{B}$ particles are picked up in the summation. In this function, the contribution from the $\boldsymbol{B} \boldsymbol{L}$ particles is less than $1 \%$ at any $T$ (so $\mathcal{B}_{j}$ may be replaced by $\mathcal{B}_{j}^{<}$in the right hand side of Eq.(3.17)). In (a) and (b) of Fig.5, $F_{s}(q, t)$ and $F_{s}^{b}(q, t)$ are compared at $T=0.24$ and 0.28 . In (c), the $T$ dependence of $F_{s}^{b}\left(q, \tau_{\alpha}\right)$ is shown at $t=\tau_{\alpha}$. It is about $30 \%$ of $F_{s}\left(q, \tau_{\alpha}\right)=e^{-1}$ for relatively high $T$, but it increases up to $80 \%$ for $T=0.24$. This dramatic change serves to slow down the relaxation of $F_{s}(q, t)$ at low $T$. In (d), we display $\tau_{\alpha}, \tau_{\mathrm{bp}}, \tau_{\mathrm{st}}$, and $\tau_{b}$, where $\tau_{\alpha}$ indeed grows most steeply with lowering $T$. Due to this crossover, we have $\tau_{b} / \tau_{\alpha} \sim 20$ for $\tau_{\alpha} \lesssim 10^{4}$ but $\tau_{b} / \tau_{\alpha} \sim 2.7$ for $\tau_{\alpha} \sim 10^{5}$ from Table 1.

\section{DIFFUSION}

\section{A. Contributions to the mean square displacement}

It is widely believed that the diffusion of a tagged or test particle in supercooled and glassy systems is caused by its jump or escape motions from temporal cages [1, 8, 11-13, 15 18]. At $T=0.24$, we show that the diffusion occurs as activation processes, where only the $\boldsymbol{B} \boldsymbol{L}$ particles can diffuse over long distances.

The mean-square displacement $M(t)$ is written as

$$
M(t)=\left\langle|\Delta r(t)|^{2}\right\rangle=\frac{1}{N} \sum_{i}\left\langle\left(\Delta r_{i}\left(t_{0}, t_{1}\right)\right)^{2}\right\rangle
$$

where $\Delta r_{i}\left(t_{0}, t_{1}\right)$ is the displacement length in Eq.(3.6) in time interval with width $t=t_{1}-t_{0}$. As is well known, $M(t)$ exhibits the ballistic behavior $\left(\propto t^{2}\right)$ in the very early stage $(t \lesssim 1)$, the plateau behavior $M(t) \cong M_{\mathrm{p}}$ in an intermediate time range, and the linear growth $M(t) \cong 6 D t$ in the late stage. In $M(t)$, the diffusion regime starts after dominance of the jump motions over the thermal vibrational motions. Since we take the average over all the particles in Eq.(4.1), $D$ in this paper is the following mean value,

$$
D=(1-c) D_{1}+c D_{2},
$$

where $D_{1}$ and $D_{2}$ are the diffusion constants of the first and second species, respectively, and $c$ is the composition.

In Fig.6a, the top curve represents $M(t)$, which exhibits its typical behaviors mentioned above with $M_{\mathrm{p}} \cong$ 

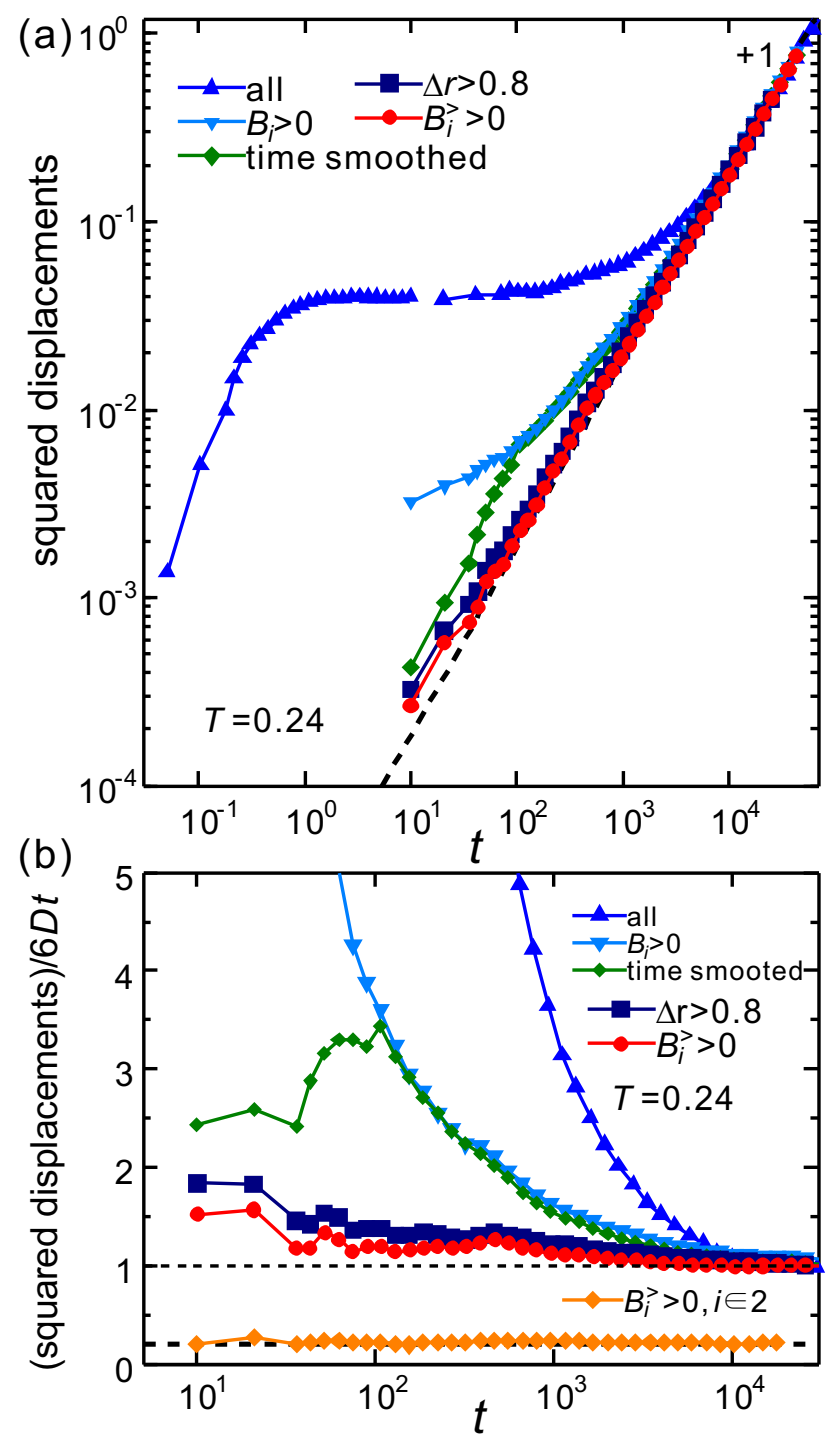

FIG. 6: (Color online) Contributions to the mean square displacement for $T=0.24$. (a) $M(t)$ from all the particles (top curve), $M_{B}(t)$ from $\boldsymbol{B}$ particles, $M_{B}^{>}(t)$ from $\boldsymbol{B} \boldsymbol{L}$ particles, $M^{>}(t)$ from those with $\Delta r_{i}>0.8$, and $\bar{M}(t)$ for timesmoothed positions on a logarithmic scale. See Eqs.(4.4)(4.7). (b) Top five curves represent contributions divided by $6 D t$ on a semi-logarithmic scale, tending to unity with increasing $t$. The approach is very rapid for $M^{>}(t) / 6 D t$ and $M_{B}^{>}(t) / 6 D t$. Shown also is the contribution divided by $6 D t$ from $\boldsymbol{B} \boldsymbol{L}$ particles belonging to the second species (bottom line), which is very close to $D_{2} /\left(D_{1}+D_{2}\right) \cong 0.2$ for $t \gtrsim 10$.

0.036. Other three curves represent the following contributions,

$$
\begin{aligned}
M_{B}(t) & =\frac{1}{N} \sum_{i}\left\langle\left(\Delta r_{i}\right)^{2} \theta\left(\mathcal{B}_{i}\right)\right\rangle, \\
M_{B}^{>}(t) & =\frac{1}{N} \sum_{i}\left\langle\left(\Delta r_{i}\right)^{2} \theta\left(\mathcal{B}_{i}^{>}\right)\right\rangle, \\
M^{>}(t) & =\frac{1}{N} \sum_{i}\left\langle\left(\Delta r_{i}\right)^{2} \theta\left(\Delta r_{i}-\ell_{\mathrm{m}}\right)\right\rangle,
\end{aligned}
$$
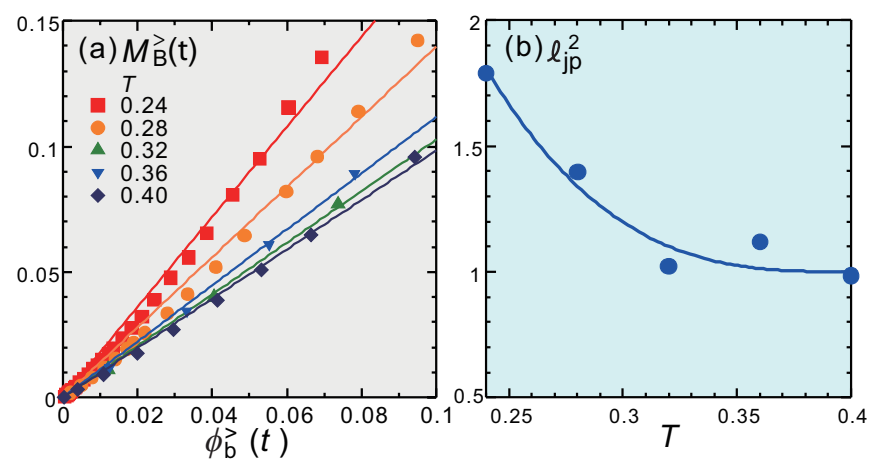

FIG. 7: (Color online) (a) $M_{B}^{>}(t)$ vs $\phi_{b}^{>}(t)$ for various $T$, where both grow linearly in time in the early stage. (b) Ratio $\ell_{\mathrm{jp}}^{2}=M_{B}^{>}(t) / \phi_{b}^{>}(t)$ vs $T$, where $\ell_{\mathrm{jp}}$ is a characteristic jump length of a particle.

where $\Delta r_{i}, \mathcal{B}_{i}$, and $\mathcal{B}_{i}^{>}$are abbreviations of $\Delta r_{i}\left(t_{0}, t_{1}\right)$, $\mathcal{B}_{i}\left(t_{0}, t_{1}\right)$, and $\mathcal{B}_{i}^{>}\left(t_{0}, t_{1}\right)$, respectively, and $\ell_{\mathrm{m}}=0.8$. Here, owing to the step function $\theta(\cdots), M_{B}(t)$ arises from the $\boldsymbol{B}$ particles, $M_{B}^{>}(t)$ from the $\boldsymbol{B} \boldsymbol{L}$ particles, and $M^{>}(t)$ from the particles with $\Delta r_{i}>0.8$. In addition, defining the smoothed position

$$
\overline{\boldsymbol{r}}_{i}(t)=\frac{1}{\Delta t} \int_{t-\Delta t}^{t} d t^{\prime} \boldsymbol{r}_{i}\left(t^{\prime}\right)
$$

with $\Delta t=100$, we calculate the time-smoothed mean square displacement,

$$
\bar{M}(t)=\frac{1}{N} \sum_{i}\left\langle\left|\overline{\boldsymbol{r}}_{i}\left(t_{0}+t\right)-\overline{\boldsymbol{r}}_{i}\left(t_{0}\right)\right|^{2}\right\rangle .
$$

In Fig.6, we can see the sequence $M_{B}(t)>\bar{M}(t)>$ $M^{>}(t)>M_{B}^{>}(t)$. To see the approach to the diffusion behavior, in Fig.6b, we display $M(t) / 6 D t, M_{B}(t) / 6 D t$, $\bar{M}(t) / 6 D t, \quad M^{>}(t) / 6 D t$, and $M_{B}^{>}(t) / 6 D t$ with $D=$ $0.596 \times 10^{-5}$. The latter four quantities approach unity more rapidly than $M(t) / 6 D t$. In particular, the jump contributions $M^{>}(t)$ and $M_{B}(t)^{>}$become very close to $6 D t$ from the very early stage $t \gtrsim 50 \sim 5 \times 10^{-4} \tau_{\alpha}$.

To be precise, we should calculate $D_{1}$ and $D_{2}$ separately. To this end, we also consider the contribution from the $\boldsymbol{B} \boldsymbol{L}$ particles belonging to the second species,

$$
M_{B 2}^{>}(t)=\frac{1}{N} \sum_{i \in 2}\left\langle\left(\Delta r_{i}\right)^{2} \theta\left(\mathcal{B}_{i}^{>}\right)\right\rangle .
$$

In Fig.6b, the ratio $M_{B 2}^{>}(t) / 6 D t$ is plotted as a flat line at the bottom. It is equal to 0.21 for $t \gtrsim 10 \sim 10^{-4} \tau_{\alpha}$. This value should be equal to $c D_{2} / D=D_{2} /\left(D_{1}+D_{2}\right)$, so we have the diffusion constant ratio $D_{2} / D_{1} \cong 0.27$ in this case. In accord with this ratio, the composition of the large particles among the $\boldsymbol{B} \boldsymbol{L}$ particles is $c_{b}^{>}(t) \cong 0.25$ for $T=0.24$ in Table 1 .

Recall that the fraction of the $\boldsymbol{B} \boldsymbol{L}$ particles $\phi_{b}^{>}(t)$ grows linearly in time in the early stage in Fig.3, leading 
to the characteristic time $\tau_{\text {st }}$ in Eq.(3.12). We may define a characteristic jump length $\ell_{\text {jp }}$ from the following ratio,

$$
\ell_{\mathrm{jp}}^{2}=M_{B}^{>}(t) / \phi_{b}^{>}(t) .
$$

Now $D$ is expressed as

$$
D=\ell_{\mathrm{jp}}^{2} / 6 \tau_{\mathrm{st}}
$$

In Fig.7a, we plot $\phi_{b}^{>}(t)$ vs $M_{B}^{>}(t)$ to show their linear relationship. In Fig.7b, we plot $\ell_{\mathrm{jp}}^{2}$ vs $T$, where $\ell_{\mathrm{jp}}$ is close to the particle diameter $\sigma_{1}$, only weakly depending on $T$. As a natural result, its $T$-dependence is similar to that of the average displacement length $\langle r\rangle_{B L}$ in Fig.4b. In our calculation of $\phi_{b}^{>}(t)$ and $M_{B}^{>}(t)$, the lower bound of the jump motions $\ell_{\mathrm{m}}$ is set equal to 0.8 for any $T$ (see Subsec.IVD for comments on this aspect).

Previously [8, 15], $M(t)$ was divided into two contributions from appropriately defined mobile and immobile particles, where the former turned out to dominate over the latter in the diffusion regime, in accord with the results in this section.

\section{B. Violation of the Stokes-Einstein relation}

In our model particle system, the violation of the Stokes-Einstein relation is ascribed to the steep growth of $\tau_{\alpha}$ at low $T$, which has been discussed in Sec.IIIC. However, we might need to include other elements (such as the nonsphericity of the particles) to explain the observed diffusion behavior in real molecular systems [5, 11, 12.

In Fig.8a, we plot $D=\left(D_{1}+D_{2}\right) / 2, D_{1}$, and $D_{2}$ vs $T$. For $T \geq 0.28$ we calculated them from the usual mean square displacements. Here, $D_{2} / D_{1} \cong 0.27$ for $T=0.24$ and 0.71 for $T=0.40$. Thus, $D_{2}$ decreases more rapidly than $D_{1}$ with lowering $T$, which is consistent with the $T$ dependence of the composition of the $\boldsymbol{B} \boldsymbol{L}$ particles in Table 1. In Fig.8b, we plot the products $D \tau_{\alpha}, D_{1} \tau_{\alpha}$, $D_{2} \tau_{\alpha}$, and $D \tau_{b}$ vs $T$. The behavior of $D_{1} \tau_{\alpha}$ is nearly the same as in the previous simulation 15]. However, $D \tau_{b}$ depends on $T$ much more weakly than $D \tau_{\alpha}$, where $\tau_{b}$ is the life time of bonds in Eq.(3.4). From Eq.(4.10) and Fig. $7 \mathrm{~b}$, we notice that the relation $\tau_{\text {st }} \cong \tau_{b} / 3$ well holds in the temperature range studied. These results indicate that the diffusion is governed by the activated dynamics, while the stress relaxation is more sensitive to the thermal fluctuations in fragile systems.

From Fig.8b, the violation of the Stokes-Einstein relation is weaker for a large particle than for a small particle. The participation of larger particles in the jump motions should become increasingly infrequent with lowering $T$, as suggested by the previous experiments [11, 12].

\section{Van Hove self-correlation function}

The time-correlation function $F_{s}(q, t)$ in Eq.(2.4) has been used so far, though it has been calculated only for

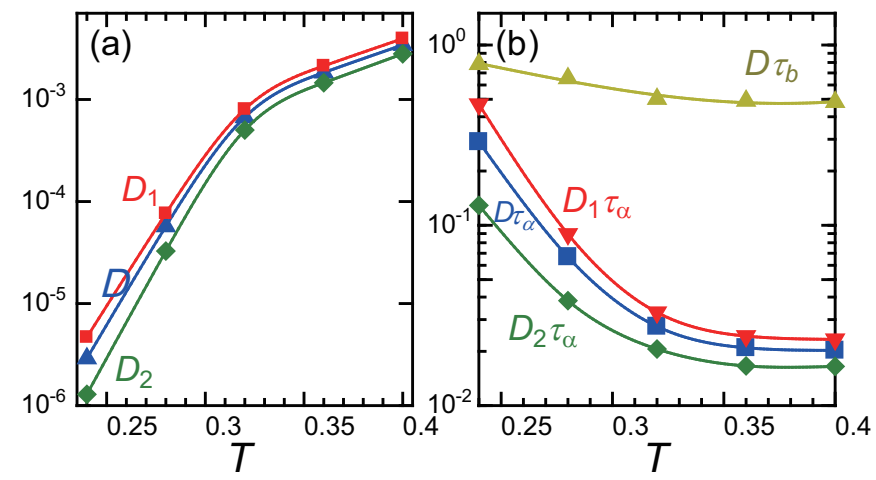

FIG. 8: (Color online) (a) Diffusion constants $D_{1}$ for small particles, $D_{2}$ for large particles, and $D=\left(D_{1}+D_{2}\right) / 2$ as functions of $T$. (b) $D_{1} \tau_{\alpha}, D_{2} \tau_{\alpha}$, and $D \tau_{\alpha}$, and $D \tau_{b}$ as functions of $T$. The curves of $D_{1} \tau_{\alpha}$ and $D_{2} \tau_{\alpha}$ indicate the violation of the Stokes-Einstein relation from $\eta / T \propto \tau_{\alpha}$, whereas $D \tau_{b}$ only weakly depends on $T$.

$q=2 \pi$. Its inverse Fourier transformation $G_{s}(r, t)$, called the van Hove self-correlation function, exhibits thermal activation behavior [14] as a function of $r=|\boldsymbol{r}|$ and $t$ as $T$ is lowered.

From Eq.(2.4) we obtain

$$
G_{s}(r, t)=\frac{1}{N} \sum_{j}\left\langle\delta\left(\Delta \boldsymbol{r}_{j}\left(t_{0}, t_{0}+t\right)-\boldsymbol{r}\right)\right\rangle,
$$

which is normalized as $4 \pi \int_{0}^{\infty} d r r^{2} G_{s}(r, t)=1$. It represents the probability of finding a particle with displacement $\boldsymbol{r}$ after time $t$. In our definition, the average of $\left\langle\delta\left(\Delta \boldsymbol{r}_{j}\left(t_{0}, t_{0}+t\right)-\boldsymbol{r}\right)\right\rangle$ is taken over all the particles, so it is the mean of the van Hove self-correlation functions for the two species as

$$
G_{s}(r, t)=(1-c) G_{s 1}(r, t)+c G_{s 2}(r, t) .
$$

Here, $G_{s \alpha}(r, t)(\alpha=1,2)$ are the averages over the particles of the species $\alpha$ with $N^{-1} \sum_{j}$ in Eq.(4.11) being replaced by $N_{\alpha}^{-1} \sum_{j \in \alpha}$. The mean square displacement $M(t)$ in Eq.(4.1) is expressed as

$$
M(t)=4 \pi \int_{0}^{\infty} d r r^{4} G_{s}(r, t) .
$$

The $M^{>}(t)$ in Eq.(4.5) follows if the lower bound of the above integral is increased to 0.8 .

In Fig. 9, we display $4 \pi r^{4} G_{s}(r, t)$ vs $r$ at various $t$. The area below its curve is equal to $M(t)$. For $T=0.24$ in (a), it exhibits a deep minimum at $r=r_{\mathrm{m}} \sim 0.7$. In the interior $r<r_{\mathrm{m}}$ it is nearly stationary, while in the exterior $r>r_{\mathrm{m}}$ it grows with two or three maxima arising from multiple jumps. From Fig.6, the area of the outer region is nearly equal to $6 D t$ for each curve. For $T=0.28$ in (b), in contrast, a shoulder first appears to grow into a peak in the exterior $r>r_{\mathrm{m}}$. The minimum at $r=r_{\mathrm{m}}$ increases considerably in time. 

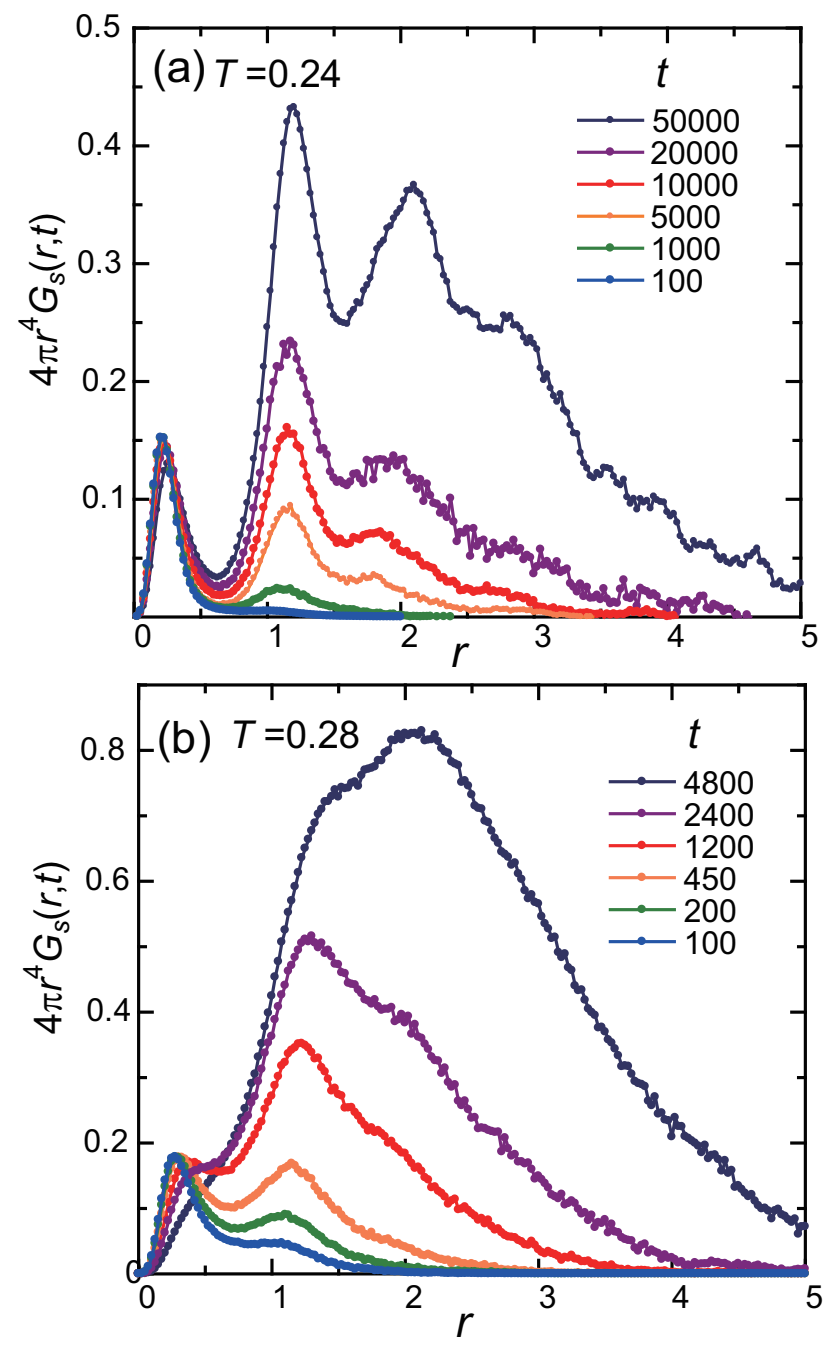

FIG. 9: (Color online) $4 \pi r^{4} G_{s}(r, t)$ vs $r$ at various $t$. (a) For $T=0.24$, there is a deep minimum at $r=r_{\mathrm{m}} \sim 0.7$, which separates a nearly stationary part for $r<r_{\mathrm{m}}$ and a growing part with multiple maxima for $r>r_{\mathrm{m}}$. (b) For $T=$ 0.28 , a minimum at $r=r_{\mathrm{m}} \sim 0.7$ increases in time with one maximum for $r>r_{\mathrm{m}}$. A secondary peak in the outer region $r>r_{\mathrm{m}}$ starts to appear as a shoulder.

From Eq.(4.12), $G_{s}(r, t)$ is equal to the mean $G_{s 1}(r, t) / 2+G_{s 2}(r, t) / 2$ for $c=1 / 2$. We are also interested in the difference of the motions of the small and large particles. Therefore, in Fig.10, we plot the three curves of $4 \pi r^{4} G_{s}(r, t), 2 \pi r^{4} G_{s 1}(r, t)$, and $2 \pi r^{4} G_{s 2}(r, t)$ at $t=10^{4}$ for $T=0.24$. All these curves exhibit a deep minimum at the common length $r=r_{\mathrm{m}}$. Because $D_{2} / D_{1}=0.27$ here, the area of $2 \pi r^{4} G_{s 1}(r, t)$ in the exterior $r>r_{\mathrm{m}}$ is four times larger than that of $2 \pi r^{4} G_{s 2}(r, t)$. A second peak corresponding to two jump motions can be seen for the small particle but not for the the large particle, although the large particles also undergo multiple jumps on long timescales.

In Fig.11, we furthermore divide $G_{s}(r, t)$ into the contributions from $\mathcal{B}_{i}\left(t_{0}, t_{0}+t\right)=k$ with $k=0,1, \cdots$ for

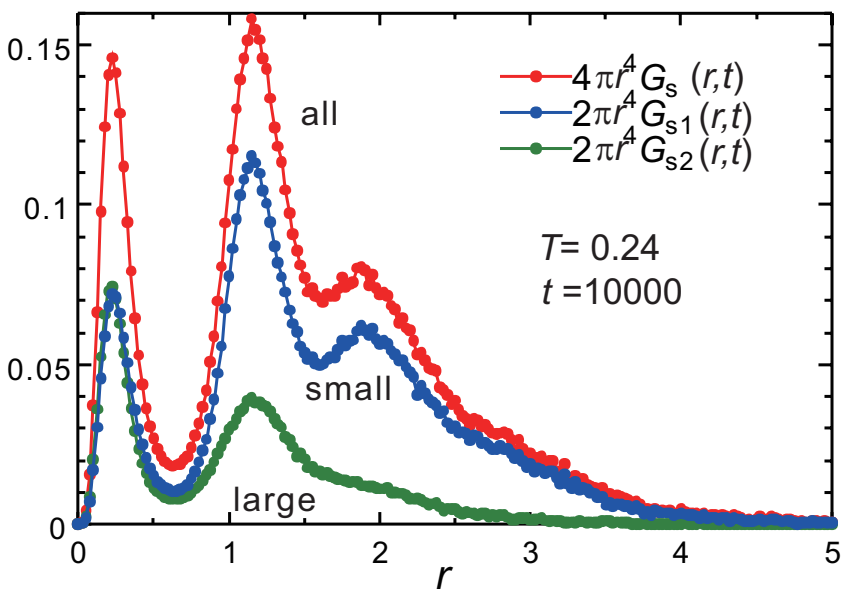

FIG. 10: (Color online) $4 \pi r^{4} G_{s}(r, t)$ is decomposed into the small particle part $2 \pi r^{4} G_{s 1}(r, t)$ and the large particle part $2 \pi r^{4} G_{s 2}(r, t)$ from Eq.(4.12) at $t=10^{4}$ for $T=0.24$. The minimum at $r=r_{\mathrm{m}}$ is common for the small and large particles.

three times. The particles with $k>4$ have undergone multiple jumps. In (a) at $t=500$, those with $k \leq 4$ have undergone a single jump, while those with $k>4$ two jumps. In (b) at $t=10^{4}$, the fraction of the particles with $k>4$ is increased. In (c) at $t=5 \times 10^{4}$, the fraction of three jumps becomes noticeable yielding a small third peak. We can thus see how multiple stringlike jump motions give rise to these contributions.

Previously, for the Lennard-Jones potential, Sastry et al. 14 and Wahnström [13 found secondary peaks in $4 \pi r^{2} G_{s}(r, t)$ at low $T$. For hard-sphere binary mixtures [16 18], the same behavior was noticed for sufficiently large volume fractions of the particles. In particular, Reichman et al. 16. found appearance of two peaks in the outer region $r>r_{\mathrm{m}}$ indicating discrete step motions of hard spheres.

The behaviors of $G_{s}(r, t)$ at $T=0.24$ can be expected generally for glassy particle systems at low $T$. A particle in such systems is analogous to a Brownian particle slowly escaping from a cage represented by a potential $U(r)$. If the barrier height $U_{\mathrm{m}}=U\left(r_{\mathrm{m}}\right)$ at $r=r_{\mathrm{m}}$ is much higher than $k_{B} T$, the probability of staying around this barrier is very small $\left(\propto \exp \left[-U_{\mathrm{m}} / k_{B} T\right]\right)$ and the probability of escaping to the outer region is proportional to $t$. The same behaviors have indeed been found for $G_{s}(r, t)$ at $T=0.24$ in Fig.9a, where the minimum length $r_{\mathrm{m}}$ is analogous to the critical radius in metastable systems.

\section{Dependence on the cut-off length $\ell_{\mathrm{m}}$}

In this paper, the lower bound $\ell_{\mathrm{m}}$ of the jump motions in Eqs.(3.7) and (3.8) has been set equal to 0.8. As remarked below Eq.(3.16), this length is between the average displacement lengths $\langle\Delta r\rangle_{B L}$ and $\langle\Delta r\rangle_{B L}$ for the $\boldsymbol{B} \boldsymbol{L}$ and $\boldsymbol{B} \boldsymbol{S}$ particles in Eqs.(3.15) and (3.16), where 

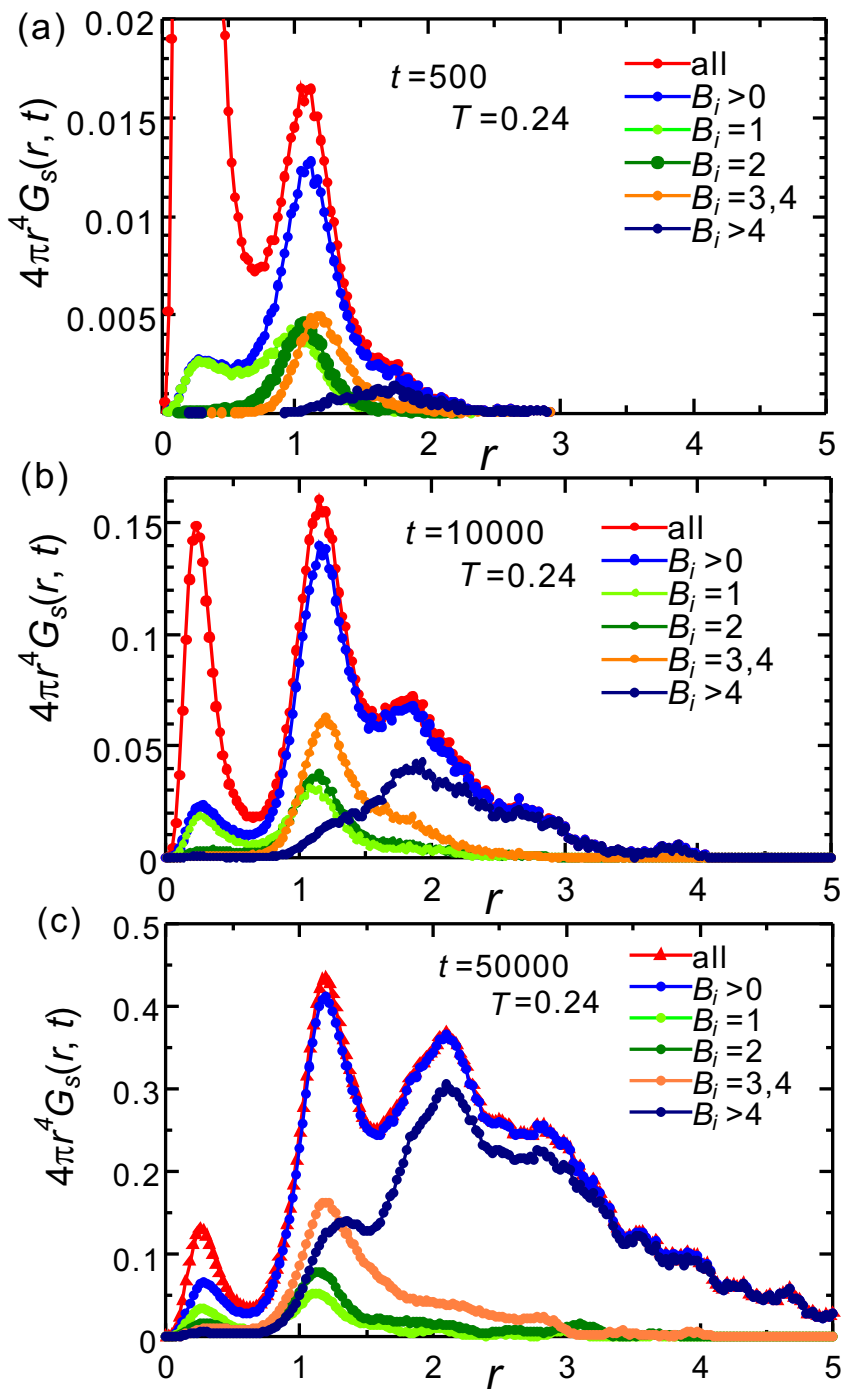

FIG. 11: (Color online) $4 \pi r^{4} G_{s}(r, t)$ vs $r$ at (a) $t=500$, (b) 10000, and (c) 50000 for $T=0.24$. The contributions from the particles with $\mathcal{B}_{i}\left(t_{0}, t_{0}+t\right)=k$ are shown, which have $k$ broken bonds in time interval $\left[t_{0}, t_{0}+t\right]$.

$\langle\Delta r\rangle_{B L}>1$ and $\langle\Delta r\rangle_{B L}<0.5$ in Fig.4b. We also note that this cut-off length is close to the minimum distance $r_{\mathrm{m}} \sim 0.7$ in $4 \pi r^{4} G_{s}(r, t)$ in Fig.9a. In Fig.12, by setting $\ell_{\mathrm{m}}=0,7,0.8$, and 0.9 , we plot the three corresponding curves of $M_{B}^{>}(t) / 6 D t$. They surely tend to unity for $t \gtrsim 10^{3}$, so $M_{B}^{>}(t)$ is insensitive to a small change of $\ell_{\mathrm{m}}$.

At $T$ higher than 0.3 , however, the cage life time becomes shorter. As a result, $\phi_{B}^{>}(t)$ in Eq.(3.10), $\tau_{\mathrm{st}}$ in Eq.(3.12), and $\ell_{\text {jp }}$ in Eq.(4.9) significantly depend on $\ell_{\mathrm{m}}$, although we have set $\ell_{\mathrm{m}}=0.8$ for any $T$.

\section{SUMMARY AND REMARKS}

In this paper, simulations have been performed on a high-density binary mixture at low $T$, where the particles interact via the soft-core potential (2.1) with

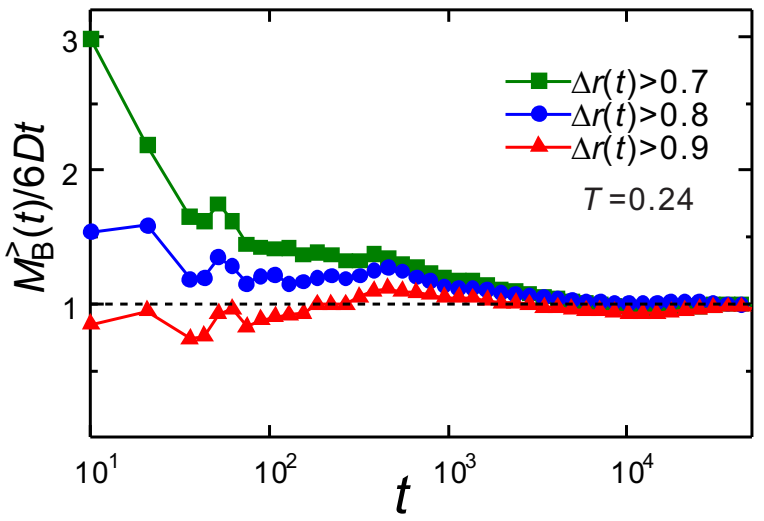

FIG. 12: (Color online) $M_{B}^{>}(t) / 6 D t$ from the $\boldsymbol{B} \boldsymbol{L}$ particles with $r>\ell_{\mathrm{m}}=0,7,0.8$, and 0.9 for $T=0.24$, where $\ell_{\mathrm{m}}=$ 0.8 in the other figures in this paper. They are close to the minimum distance $r_{\mathrm{m}} \sim 0.7$ of $4 \pi r^{4} G(r, s)$ in Fig.9a. These three curves converge to unity for $t \gtrsim 1000$.

the size ratio $\sigma_{2} / \sigma_{1}=1.2$. The total particle number $N$ is $10^{4}$ and the density $n$ is $0.8 \sigma_{1}^{-3}$. Our main results are summarized as follows.

(i) In Sec.III, using the broken bond number $\mathcal{B}_{i}\left(t_{0}, t_{1}\right)$ in Eq.(3.5), we have visualized the particles composing strings and those surrounding them in Fig.1. The former have undergone large displacements $\left(\Delta r_{i}>0.8\right)$ with multiple broken bonds $\left(\mathcal{B}_{i}>1\right)$, while the latter small displacements $\left(\Delta r_{i}<0.8\right)$ with a single broken bond $\left(\mathcal{B}_{i}=1\right)$. The number of the latter is several times larger than that of the former because of the large coordination numbers in $3 \mathrm{D}$, which leads to the difference of the fraction of the former $\phi_{b}^{>}(t)$ and that of the latter $\phi_{b}^{<}(t)=\phi_{b}(t)-\phi_{b}^{>}(t)$ as in Fig.3. These two kinds of motions become increasingly distinct as $T$ is lowered as in Figs.1, 2, and 4 and in Table 1. The latter contribution to $F_{s}(q, t)$ in Eq.(2.4) becomes long-lived at low $T$ as illustrated in Fig.5. This leads to the steeper rise of $\tau_{\alpha}$ than the other characteristic times related to the bond breakage at low $T$ in Fig.5d.

(ii) In Sec.IV, we have found that the contribution to the mean square displacement $M(t)$ from the particles with large displacements behaves as $6 D t$ soon after the ballistic regime. The origin of the violation of the Stokes-Einstein relation has been ascribed to the steep growth of $\tau_{\alpha}(\propto \eta)$ at low $T$ for our system.

We further give some remarks in the following. (1) In our fragile glass-former, the non-Arrhenius behavior in the Angell plot (the steep growth in the curve of $\log \eta$ vs $1 / T$ ) [2] and the violation of the Stokes-Einstein relation [11, 12 are closely related under the condition $\tau_{\alpha} \sim \eta / T$. Note that we have not calculated $\eta$ but assumed $\eta \propto T \tau_{\alpha}$ from Eq.(2.6). In future, we should calculate $\eta$ together with $D$ for lower $T$.

(2) In a 50:50 binary mixture with the soft-core potential, we have examined the particle motions around strings. We should examine whether our results remain valid or 
need to be modified for other compositions and for other particle interactions [16, 23]. We should note that a 20:80 mixture with a Lennard-Jones potential was used to detect strings in the original work [4. Stringlike collective motions were also observed in polycrystalline systems numerically [24, 25] and experimentally [26].

(3) The diffusion behavior of the mean square displacement $M(t)$ can be obtained soon after the ballistic regime if the contribution from large displacements are picked up. This is a natural result in the activated dynamics. For lower $T$, the diffusion constant $D$ is obtainable in this manner, where its aging behavior will be of interest.

\section{Acknowledgments}

This work was supported by Grant-in-Aid for Scientific Research from the Ministry of Education, Culture, Sports, Science and Technology of Japan. T. K. was supported by the Japan Society for Promotion of Science. The authors would like to thank Hayato Shiba, Kunimasa Miyazaki, and Kang Kim for informative discussions. The numerical calculations were carried out on SR16000 at YITP in Kyoto University.
[1] K. Binder and W. Kob, Glassy Materials and Disordered Solids (World Scientific, Singapore, 2005).

[2] C. A. Angel, J. Phys. Chem. Sol. 49, 863 (1988).

[3] H. R. Schober, C. Oligschleger, and B. B. Laird, J. NonCryst. Solids 156-158, 965 (1993); C. Oligschleger and H. R. Schober, Phys. Rev. B 59, 811 (1999).

[4] W. Kob, C. Donati, S. J. Plimton, P. H. Poole, and S. C. Glotzer, Phys. Rev. Lett. 79, 2827 (1997); C. Donati, J. F. Douglas, W. Kob, S. J. Plimton, P. H. Poole, and S. C. Glotzer, Phys. Rev. Lett. 80, 2338 (1998); C. Donati, S. C. Glotzer, P. H. Poole, W. Kob, and S. J. Plimpton, Phys. Rev. E 60, 3107 (1999).

[5] S. C. Glotzer, J. Non-Cryst. Solids 274, 342 (2000).

[6] R. Yamamoto and A. Onuki, J. Phys. Soc. Jpn., 66, 2545 (1997).

[7] R. Yamamoto and A. Onuki, Phys. Rev. E 58, 3515 (1998); R. Yamamoto and A. Onuki, J. Phys.: Condens. Matter 29, 6323 (2000).

[8] M. M. Hurley and P. Harrowell, Phys. Rev. E 52, 1694 (1995); D.N. Perera, J. Phys.: Condens. Matter 10, 10115 (1998).

[9] L. Berthier, G. Biroli, J.-P. Bouchaud, L. Cipelletti, and W. van Saarloos, Dynamical Heterogeneities in Glasses, Colloids, and Granular Media (Oxford University Press, Oxford, 2011).

[10] H. Shiba, T. Kawasaki, and A. Onuki, Phys. Rev. E 86, 041504 (2012).

[11] I. Chang, F. Fujara, B. Geil, G. Heuberger, T. Mangel, H. Sillescu, J. Non-Cryst. Solids 172-174, 248 (1994); H. Sillescu, J. Non-Cryst. Solids 243, 81 (1999).
[12] M.T. Cicerone, F.R. Blackburn and M.D. Ediger, Macromolecules 28, 8224 (1995); M. D. Ediger, Annu. Rev. Phys. Chem. 51, 99 (2000).

[13] G. Wahnström, Phys. Rev. A 44, 3752 (1991).

[14] S. Sastry, P. G. Debenedetti, and F. H. Stillinger, Nature 393, 555 (1998).

[15] R. Yamamoto and A. Onuki, Phys. Rev. Lett. 81, 4915 (1998).

[16] D. Reichman, E. Rabani, and P. Geissler, J. Phys. Chem. B 109, 14654 (2005).

[17] E. J. Saltzman and K. S. Schweizer, Phys. Rev. E 77, 051504 (2008).

[18] E. Flenner, M. Zhang, and G. Szamel, Phys. Rev. E 83, 051501 (2011).

[19] A. Furukawa and H. Tanaka, Phys. Rev. E 84, 061503 (2011).

[20] H. Shiba and A. Onuki, Phys. Rev. E 81, 051501 (2010).

[21] K. Vollmayr-Lee, J. Chem. Phys. 121, 4781 (2004)

[22] A. Onuki, Phase Transition Dynamics, (Cambridge University Press, Cambridge, 2002).

[23] F. W. Starr and J. F. Douglas, Phys. Rev. Lett. 106, 115702 (2011); Z. Zhang, P. J. Yunker, P. Habdas, and A. G. Yodh, Phys. Rev. Lett. 107, 208303 (2011).

[24] T. Hamanaka and A. Onuki, Phys. Rev. E 74, 011506 (2006); ibid. 75, 041503 (2007).

[25] H. Zhang, D. J. Srolovitz, J. F. Douglas, and J. A. Warren, PNAS 106 May 12, 7735 (2009).

[26] C. R. Berard, K. Barros, J. F. Douglas, and W. Losert, Phys. Rev. E 81, 041301 (2010). 